\title{
Tiers garant - Segen oder Fluch?
}

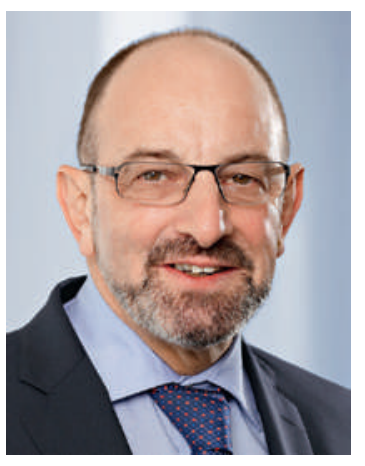

Auf die Frage, welches Abrechnungsverfahren im ambulanten Bereich das «richtige» sei, gibt es seit der Einführung des TARMED verschiedene Antworten. Die FMH hat die Thematik neu aufgegriffen und eine Auslegeordnung der Vor- und Nachteile bestehender Systeme erstellt. Die Ergebnisse sind in ein Positionspapier der FMH vom Januar 2014 eingeflossen: Aus triftigen Gründen empfiehlt die FMH im ambulanten Bereich weiterhin den Tiers garant als Regelfall.

Verschiedene Aussagen von Kolleginnen und Kollegen sowie Leserbriefe auf das Positionspapier der FMH zeigen, dass die verschiedenen Abrechnungsverfahren unterschiedlich beurteilt werden. Dies mag einerseits darin begründet sein, dass beim Entscheid für den Tiers payant Bequemlichkeit, ein vermeintliches geringeres Inkassorisiko und mangelnder Informationsstand eine grosse Rolle spielen. Andererseits stehen beim Tiers garant das Patientenwohl (z. B. Datenschutz, Rechnungskopie) sowie die eigenen Daten im Zentrum, die nur über den Kanal des Tiers garant lückenlos sind und auch die Out-of-Pocket-Zahlen beinhalten.

Selbstverständlich können ambulant tätige Ärztinnen und Ärzte weiterhin ihr bevorzugtes Abrechnungssystem wählen. Eingeschränkt wird ihre Wahlfreiheit aber durch das KVG und den Rahmenvertrag, dem sie sich angeschlossen haben: Denn sowohl das Gesetz als auch der Rahmenvertrag machen den Tiers garant zum Regelfall und den Tiers payant zur Ausnahme. Auch wenn dem Tiers garant damit der Vorzug gegeben wird, bleibt die Wahlfreiheit bestehen: Weder ist der Tiers payant für die Ärzteschaft deletär, noch wird die Frage Tiers payant oder Tiers garant zum Lackmustest für die Verbandstreue.

\section{Im Abrechnungssystem des Tiers garant steht der Patient im Mittelpunkt ...}

Für mich als Tarifverantwortlicher steht nicht der Tiers payant / Tiers garant als Pièce de Résistance, sondern vielmehr das Bereitstellen der eigenen Daten im Vordergrund. Ohne ärzteeigene Datengrundlage wären die Arbeiten beim Point-of-Care-Tarif im Praxislabor, beim margenfreien Mo- dell der ärztlichen Medikamentenabgabe und der verschiedenen Fachteams innerhalb der Gesamtrevision der Tarifstruktur TARMED nicht möglich und ein Abschluss der Arbeiten bis Ende 2015 in weiter Ferne. Der Abdeckungsgrad ist zwar generell gut bis sehr gut, aber in einigen Bereichen lässt er leider noch zu wünschen übrig. Ihre Mitarbeit ist wichtig: Stellen Sie uns Ihre Abrechnungs-Daten und Ihre Buchhaltungszahlen im Rahmen der ROKO zur Verfügung und übermitteln Sie diese an Ihr Trust Center beziehungsweise an die Ärztekasse. Herzlichen Dank für Ihr Engagement.

\section{... er kann die Rechnungen kontrollie- ren und entscheiden, ob seine Kranken- kasse die Diagnose einsehen kann.}

Ein wesentlicher Grund, wieso die FMH weiterhin den Tiers garant empfiehlt, ist und bleibt die Position des Patienten. Im Tiers payant erhält er - obwohl vorgeschrieben - selten eine Rechnungskopie. Somit entfällt die wichtige Rechnungskontrolle durch ihn. In diesem Zusammenhang wird gerne ins Feld geführt, dass die TARMED-Rechnung sowieso schwierig zu lesen sei und der Patient deswegen die Rechnungskontrolle nicht durchführe. Die Erfahrung zeigt hingegen, dass die Patienten ihre Rechnungen genau lesen und sehr wohl auch Fehler entdecken. Zudem ist im Tiers garant auch der Daten- und Persönlichkeitsschutz gewährleistet: Der Patient entscheidet, ob er die Rechnung einreichen will oder nicht. Eventuell möchte er nicht, dass seine Diagnosen durch die Krankenkassen einsehbar sind. Zudem kann er auch entscheiden, ob er die Rechnung selbst bezahlen will oder nicht. So gelangen viele Rechnungen nicht zu den Krankenkassen, was wiederum eine grosse Kostenersparnis bedeutet.

Beide Systeme haben ihre Vorzüge, aber auch ihre Nachteile. Sie wurden in den verschiedenen Artikeln der Schweizerischen Ärztezeitung bereits aufgeführt. Auf Seite 903 dieser Ausgabe finden Sie den in der Ärzteschaft breit abgestützten Artikel «Tiers garant oder Tiers payant?», welcher die beiden Systeme nochmals vertieft beleuchtet. Unserer Ansicht nach überwiegen die Vorteile des Tiers garant, doch auch die Abläufe im Tiers garant haben Verbesserungspotential. Packen wir es an!

Dr. med. Ernst Gähler, Vizepräsident der FMH, Departementsverantwortlicher Ambulante Tarife und Verträge Schweiz / Paramedizinische Berufe 